\title{
man \\ Characterization of Effective Diffusion within Viscoelastic Fluids with Elastic Instabilities
}

\author{
Valerie Hietsch ${ }^{1}$, Phil Ligrani ${ }^{2, *}$ and Mengying $\mathrm{Su}^{2}$ \\ 1 Institute of Aerospace Thermodynamics, University of Stuttgart, 70569 Stuttgart, Germany; \\ hietschvalerie@gmail.com \\ 2 Department of Mechanical and Aerospace Engineering, University of Alabama in Huntsville, \\ Huntsville, AL 35899, USA; mengying.su@springernature.com \\ * Correspondence: pml0006@uah.edu
}

check for updates

Citation: Hietsch, V.; Ligrani, P.; Su, M. Characterization of Effective Diffusion within Viscoelastic Fluids with Elastic Instabilities. Fluids 2022, 7, 33. https://doi.org/10.3390/ fluids7010033

Academic Editors: Robert Handler and Mahmoud Mamou

Received: 3 December 2021

Accepted: 9 January 2022

Published: 13 January 2022

Publisher's Note: MDPI stays neutral with regard to jurisdictional claims in published maps and institutional affiliations.

Copyright: (C) 2022 by the authors. Licensee MDPI, Basel, Switzerland. This article is an open access article distributed under the terms and conditions of the Creative Commons Attribution (CC BY) license (https:// creativecommons.org/licenses/by/ $4.0 /)$.

\begin{abstract}
We considered effective diffusion, characterized by magnitudes of effective diffusion coefficients, in order to quantify mass transport due to the onset and development of elastic instabilities. Effective diffusion coefficient magnitudes were determined using different analytic approaches, as they were applied to tracked visualizations of fluorescein dye front variations, as circumferential advection was imposed upon a flow environment produced using a rotating Couette flow arrangement. Effective diffusion coefficient results were provided for a range of flow shear rates, which were produced using different Couette flow rotation speeds and two different flow environment fluid depths. To visualize the flow behavior within the rotating Couette flow environment, minute amounts of fluorescein dye were injected into the center of the flow container using a syringe pump. This dye was then redistributed within the flow by radial diffusion only when no disk rotation was used, and by radial diffusion and by circumferential advection when disk rotation was present. Associated effective diffusion coefficient values, for the latter arrangement, were compared to coefficients values with no disk rotation, which were due to molecular diffusion alone, in order to quantify enhancements due to elastic instabilities. Experiments were conducted using viscoelastic fluids, which were based on a $65 \%$ sucrose solution, with different polymer concentrations ranging from $0 \mathrm{ppm}$ to $300 \mathrm{ppm}$. Associated Reynolds numbers based on the fluid depth and radially averaged maximum flow velocity ranged from 0.00 to 0.5 . The resulting effective diffusion coefficient values for different flow shear rates and polymer concentrations quantified the onset of elastic instabilities, as well as significant and dramatic changes to local mass transport magnitudes, which are associated with the further development of elastic instabilities.
\end{abstract}

Keywords: elastic instability; Weissenberg number; rotating Couette flow; non-Newtonian fluid flow; diffusive transport enhancement; effective diffusion

\section{Introduction}

Engineers are facing new challenges associated with local flow transport within microscale and millimeter-scale devices in the fields of gas turbine cooling, electronic component cooling, information technology, and medical engineering. Often required in these environments are substantial enhancements to mass, momentum, and thermal transport. However, local transport, within such small-scale systems, is generally very small, and only due to molecular Brownian motions when Newtonian fluid flows are laminar and steady. This is a consequence of low Reynolds numbers, resulting from low velocities and small dimensions. By adding dilute concentrations of high-molecular-weight polymers to Newtonian solvents, the resulting viscoelastic non-Newtonian fluid can show turbulent-like characteristics at low Reynolds numbers. Such an approach can result in the development of elastic instabilities and elastic turbulence, which induce intense local fluid motions, with enhanced local flow mixing and augmented local mass, momentum, and thermal transport. 
Such characteristics are possible even for flows with Reynolds numbers that are lower than 1 . As a consequence, non-Newtonian viscoelastic fluids with elastic instabilities and elastic turbulence are attracting attention, as they provide important advantages within micro-scale and millimeter-scale environments, compared to Newtonian fluids.

Newtonian fluids are characterized by a constant viscosity and a linear relationship between shear stress and shear rate. Non-Newtonian fluids exhibit a varying viscosity, which is dependent on shear rate. Viscoelastic polymer solutions are non-Newtonian, and for higher polymer concentrations, can have shear thinning properties. In contrast, with sufficiently dilute polymer solutions, with very small polymer concentrations, according to James [1], Boger fluids are polymer solutions for which the shear thinning is negligible and the viscosity is approximately constant as the shear rate varies. Boger fluids therefore have nearly Newtonian characteristics when no elastic turbulence is present. James [1] further stated that the elastic effects of the Boger fluid can be separated from viscous effects for relatively low shear rates. In contrast, as the shear rates increase to exceed a critical value, the viscosity increases significantly due to elastic instabilities, which are related to local transverse stress variations, such as from curved streamlines. The resulting fluid then shows nonlinear and non-Newtonian behavior as elastic instabilities are initiated and develop.

Different features of viscoelastic fluids and elastic turbulence have been investigated for various geometries and flows. For example, Groisman et al. [2] compared elastic and inertial instabilities in polymer solution flows. Groisman et al. [3] investigated the elastic turbulence in a swirling flow between two plates, Couette-Taylor flow between two cylinders, and within curvilinear channels. Additional results for chaotic flows in curvilinear channel were provided by Burghelea et al. [4,5], who conducted experiments in von Kármán swirling flows. Whalley et al. [6] considered micro-scale serpentine channel flows and stated that small-scale viscoelastic flows are featured at low Reynolds numbers, and for Weissenberg numbers greater than 1.0.

Considered within the present investigation was effective diffusion, characterized by magnitudes of effective diffusion coefficients, in order to quantify mass transport due to the onset and development of elastic instabilities. Effective diffusion coefficient magnitudes were determined using different analytic approaches, as they were applied to tracked visualizations of fluorescein dye front variations, as circumferential advection was imposed upon a flow environment produced using a rotating Couette flow arrangement. Effective diffusion coefficient results were provided for a range of flow shear rates, which were produced using different Couette flow rotation speeds and two different flow environment fluid depths. Associated effective diffusion coefficient values were compared to coefficients values with no disk rotation, which were compared to molecular diffusion alone, in order to quantify enhancements due to elastic instabilities. Experiments were conducted using viscoelastic fluids, which were based on a $65 \%$ sucrose solution, with different polymer concentrations ranging from $0 \mathrm{ppm}$ to $300 \mathrm{ppm}$. Associated Reynolds numbers were based on a fluid depth range from 0.00 to 0.50 .

The results from the present investigation are unique, first, because local variations in mass species transport were quantified using measured effective diffusion variations in fluorescein dye. Second, documentation was provided of the associated transport alterations that develop from the onset and development of elastic instabilities. Of particular significance are the resulting increases in effective diffusion coefficients due to elastic instabilities, relative to flow arrangements with no instabilities, and relative to environments with molecular diffusion alone.

\section{Experimental Approach, Apparatus, and Procedures}

\subsection{Rotating Couette Flow Apparatus}

Figure 1 shows the overall concept of the rotating Couette flow arrangement with corresponding dimensions. The figure includes the coordinate systems whose origin is in the center of the bottom surface of the cup flow container. The $z$-axis is vertical to the 
bottom surface and directed upward. The r-axis is in the plane of the cup flow container bottom surface and normal to the z-axis. With this arrangement, flow motion is induced within the gap between the rotating disk $\left(R_{1}=38.1 \mathrm{~mm}\right)$ and the cup flow container surface $\left(\mathrm{R}_{2}=43.5 \mathrm{~mm}\right)$. The employed magnitude of shear rate is controlled by disk rotational speed and fluid depth. The associated shear rate $\dot{\gamma}$ is determined by

$$
\dot{\gamma}=\frac{d u}{d z}=\frac{\omega r}{H}
$$

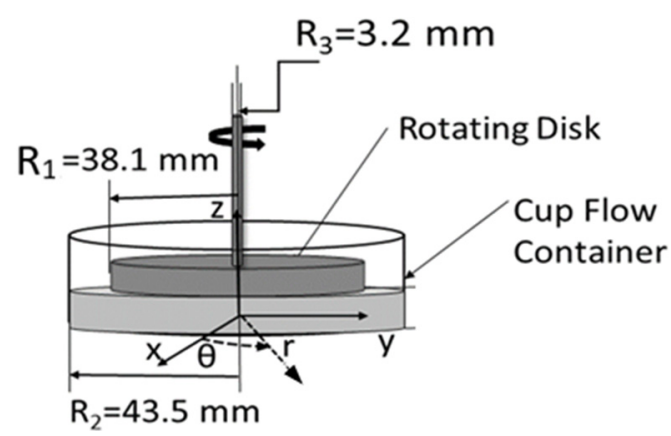

Figure 1. Coordinate systems and dimensions of rotating Couette flow device from Ligrani et al. [7] (used with copyright permission).

The shear rate is the derivative of streamwise fluid velocity $u$ with respect to the $\mathrm{z}$-coordinate, where $\omega$ is the disk rotation speed, $r$ is the radial coordinate, and $H$ is the fluid depth between the rotating disk and the cup container surface. The Reynolds number Re based upon fluid depth for each position in the flow is then given by

$$
\operatorname{Re}=\frac{\rho_{d} \omega r H}{2 \eta}
$$

where $\rho_{d}$ is the density of the fluid and $\eta$ is the viscosity, which is assumed to be constant as the shear rate varies. Here, the value of $r$ employed to determine the shear rate, and the Reynolds number is $R_{3}$. This relatively small value of $r$ is associated with minimum values of spatially varying parameters at the onset of elastic instability. The resulting Reynolds numbers range from 0.00 to 0.50 .

The PEEK plastic disk is driven by a MAXON EC32 motor, which is mounted on top of the stainless-steel assembly and controlled through the connected LABVIEW 32-bit software, version 2014-Sp1. The motor provides a power of $80 \mathrm{~W}$ and is supplied by a power supply with a $48 \mathrm{~V}$ DC supply voltage and a peak current of 12 amperes. A rotational speed can be employed in the range from 1 to 250 RPM. In order to vary the gap height, the disk is adjustable relative to the cup flow container surface. The Mitutoyo absolute depth gauge is used to measure gap height, which is specified as the distance between the cup flow container bottom surface and the disk lower surface.

\subsection{Flow Visualization Apparatus and Procedures}

Figure 2 presents a schematic diagram of a cross-sectional view of the assembled flow visualization measurement apparatus, including dimensions and components. Here, the cup flow container is transparent. A mirror is placed underneath the cup flow container with a $45^{\circ}$ angle to reflect the flow images. Note that fluid location distributions relative to the rotating disk are consistent in Figures 1 and 2 provided the fluid is opaque. A Point Gray Research Inc. CMLN-13S2C-CS USB digital camera is connected to a Dell PC computer to acquire the resulting flow visualization images. Corresponding Point Gray software is used to acquire digital images with a rate of one image per second for a period of $10 \mathrm{~min}$. The camera is connected to the Dell PC computer through a USB port. Flow visualization 
images are saved by the software as a 24-bit indexed .bmp image in a separate folder for each testing condition.

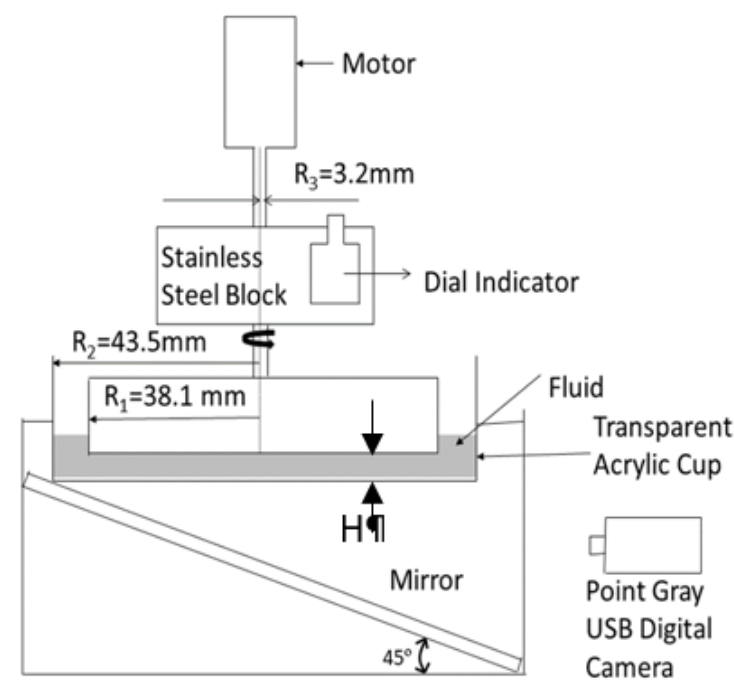

Figure 2. Arrangement and dimensions of flow visualization measurement apparatus in a crosssectional view from Ligrani et al. [7] (used with copyright permission).

To visualize local flow phenomena, a sucrose solution with red tracer dye is injected into the fluid in the cup flow container. The dye is composed of $5 \%$ of Kingscote Inc. fluorescein FWT red tracer dye mixed with sucrose solution, without any polymers present. An approximate amount of 0.05 to $0.07 \mathrm{~mL}$ of the dye solution is employed for this purpose, as it is injected into the central point of the cup flow container using a needle. This needle provides locally precise injection, as it is connected to the syringe pump by means of flexible tubing. For each experimental visualization, this needle is inserted into the cup flow container through a hole with a $0.5 \mathrm{~mm}$ diameter, which is placed in one sidewall and $2 \mathrm{~mm}$ above the bottom surface. This procedure is performed to prevent any unintended injection of small air bubbles into the flow arrangement. Afterward, the needle is removed from the flow, without causing any local flow disturbances. Then, image recording and the motor that drives the disk are started simultaneously, for each experimental condition.

\subsection{Polymer Solution Preparation and Characterization}

Within the present research effort, testing was conducted with different polymer solution concentrations ranging from $0 \mathrm{ppm}$ to $300 \mathrm{ppm}$. Pure water and a sucrose solution, both with $0 \mathrm{ppm}$ of polymers, were also tested. The sucrose solution was comprised of $65 \%$ sucrose, $34 \%$ distilled water, and 1\% sodium chloride. An appropriate amount of polymers was added to create the polymer solutions, using polyacrylamide powder from Dalton Polysciences Inc. For example, $390 \mathrm{~g}$ of sucrose, $204 \mathrm{~g}$ of distilled water, $6 \mathrm{~g}$ of $\mathrm{NaCl}$, and $0.018 \mathrm{~g}$ of polymers were combined in order to create a solution with $300 \mathrm{ppm}$ (parts per million) concentration. To generate homogenous polymer solutions, each mixture was gently stirred for four hours at a very low rotational speed, prior to testing. As solutions were prepared and investigated, extra care was employed to avoid inappropriate contaminations. Additional details are provided by Ligrani et al. [7].

Polymer mixture rheological data were obtained using a state-of-the-art commercial Anton Paar 92002 MCR 302 Modular Compact Rheometer with a sample-adaptive motor controller and normal force sensor. These data were employed to provide information regarding absolute viscosity properties of the different fluids that were used for testing at different magnitudes of shear rate. Care was employed as experiments were conducted to avoid the use of solutions after the degradation of polymer molecules occurs. Care was also taken during testing to avoid contamination by previously employed solutions. As shear rates within the present study were relatively small, the possibility of chain scission polymer 
degradation was very unlikely. In addition, the possibility of oxygen/reductive degradation was also minimized. Additional details are provided by Ligrani et al. [7].

\section{Experimental Results}

Included are time sequences of visualization images, showing fluorescein dye distributions. Diffusion coefficients, determined from these data with no advection, are provided, along with effective diffusion coefficients, determined with circumferential advection. Associated data are given for fluid depth values of $5 \mathrm{~mm}$ and $7 \mathrm{~mm}$ with no disk rotation, as well as with different disk rotation speeds. Results are given when no polymers are included, and for flows with several different polymer concentrations. Provided also are solution rheological property information, Prandtl number data for different polymer concentrations, and Schmidt number data for different polymer concentrations.

\subsection{Solution Rheological Properties}

Table 1 presents viscosity values, determined using the Anton Paar rheometer, for the sucrose and polymer solutions that are tested with different polymer concentrations $\rho$. Note that absolute viscosity values are provided for zero shear rate and for an infinite value of shear rate. Here, absolute viscosity values for zero shear rate are slightly larger than the values associated with higher, nonzero shear rates (where absolute viscosity is approximately constant with shear rate). As such, the present polymer solutions exhibit mild shear-thinning behavior as the shear rate approaches zero.

Table 1. Viscosity properties of sucrose and polymer solutions for different polymer concentrations $\rho$ for zero shear rate and for infinite value of shear rate.

\begin{tabular}{cccccc}
\hline $\begin{array}{c}\rho \\
(\mathbf{p p m})\end{array}$ & $\mathbf{0}$ & $\mathbf{8 0}$ & $\mathbf{1 0 0}$ & $\mathbf{1 5 0}$ & $\mathbf{3 0 0}$ \\
\hline$\eta_{\mathrm{o}}(\mathrm{Pa} \cdot \mathrm{s})$ & 0.187 & 0.220 & 0.287 & 0.345 & 0.439 \\
\hline$\eta_{\infty}(\mathrm{Pa} \cdot \mathrm{s})$ & - & 0.200 & 0.200 & 0.280 & 0.330 \\
\hline
\end{tabular}

The Weissenbeg number is useful for characterizing elastic instability and elastic turbulence phenomena, as expressed using

$$
W i=\lambda \dot{\gamma}
$$

Relevant also are the Deborah number and the elastic number, as given by respective equations that are of the form

$$
\begin{gathered}
D e b=\lambda \omega \\
E l=W i / R e
\end{gathered}
$$

The elastic number quantifies the relative influences of viscoelastic and viscous /inertial effects. Estimating the values of these parameters requires the determination of the polymer relaxation time $\lambda$. Within the present investigation, $\lambda$ values for different polymer concentrations from Ligrani et al. [7] are employed, which are based upon analytic procedures from Zimm [8]. For example, $\lambda=0.68 \mathrm{~s}$ for $\rho=50 \mathrm{ppm}$.

For this polymer concentration, a rheometer oscillatory frequency-sweep test is additionally used to determine the variations in viscous modulus G' and elastic modulus G' with angular frequency. Note that some sources refer to $G^{\prime \prime}$ as a loss component modulus, and $G^{\prime}$ as a storage component modulus. Note that $G^{\prime}$ is not equal to Young's modulus. The resulting data in Figure 3 indicate that the relaxation time $\lambda$ is $0.63 \mathrm{~s}$, determined from the angular frequency at the intersection value of these two moduli $[9,10]$. 


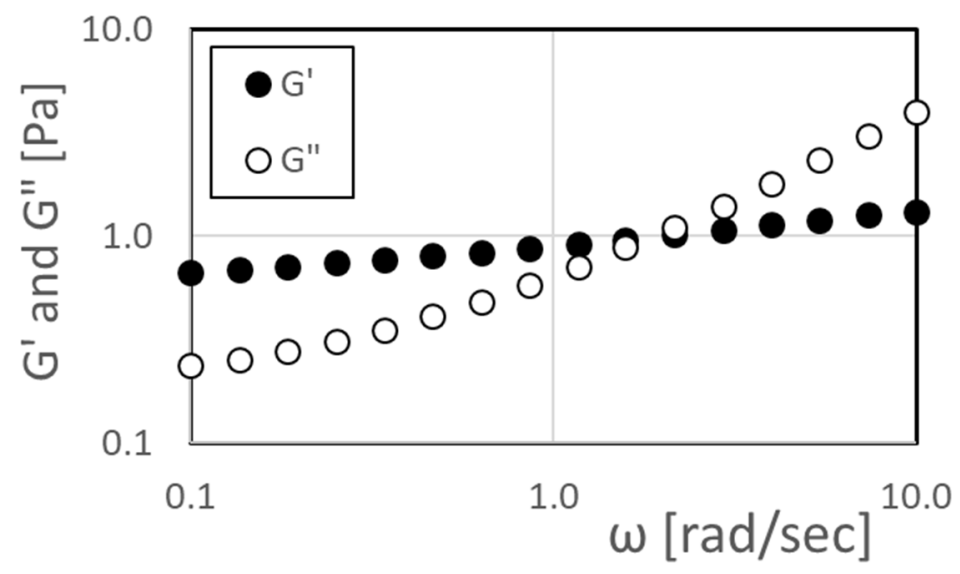

Figure 3. Viscous modulus $G^{\prime \prime}$ and elastic modulus $G^{\prime}$ variation with angular frequency from rheometer oscillatory frequency-sweep test for a solution with a polymer concentration $\rho$ of $50 \mathrm{ppm}$.

Using complex viscosity measurement procedures described by Yao et al. [11], the dependence of $\eta^{\prime \prime} /\left(\eta^{\prime} \omega\right)$ on angular frequency $\omega$ is also determined using a rheometer oscillatory frequency-sweep test. Here, $\eta^{\prime}$ is the in-phase viscosity (also referred to as the elastic portion of complex viscosity) and $\eta$ " is the out-of-phase viscosity (also referred to as the viscous portion of complex viscosity). In addition, $\eta^{\prime}=G^{\prime} / \omega$ and $\eta^{\prime \prime}=G^{\prime \prime} / \omega$. Complex viscosity is the frequency-dependent viscosity function determined for a viscoelastic fluid by subjecting it to oscillatory shear stress, produced using the rheometer oscillatory frequency-sweep test. With such an approach, phase variations can exist between the oscillatory shear stress and complex viscosity portions. Note that the magnitude of complex viscosity approaches the absolute viscosity as the frequency of oscillatory shear stress approaches zero. The resulting $\eta^{\prime \prime} /\left(\eta^{\prime} \omega\right)$ data are shown in Figure 4, which, for a polymer concentration $\rho$ of $50 \mathrm{ppm}$, are then extrapolated to a zero value of angular frequency $\omega$ to give $\lambda=0.615 \mathrm{~s}$.

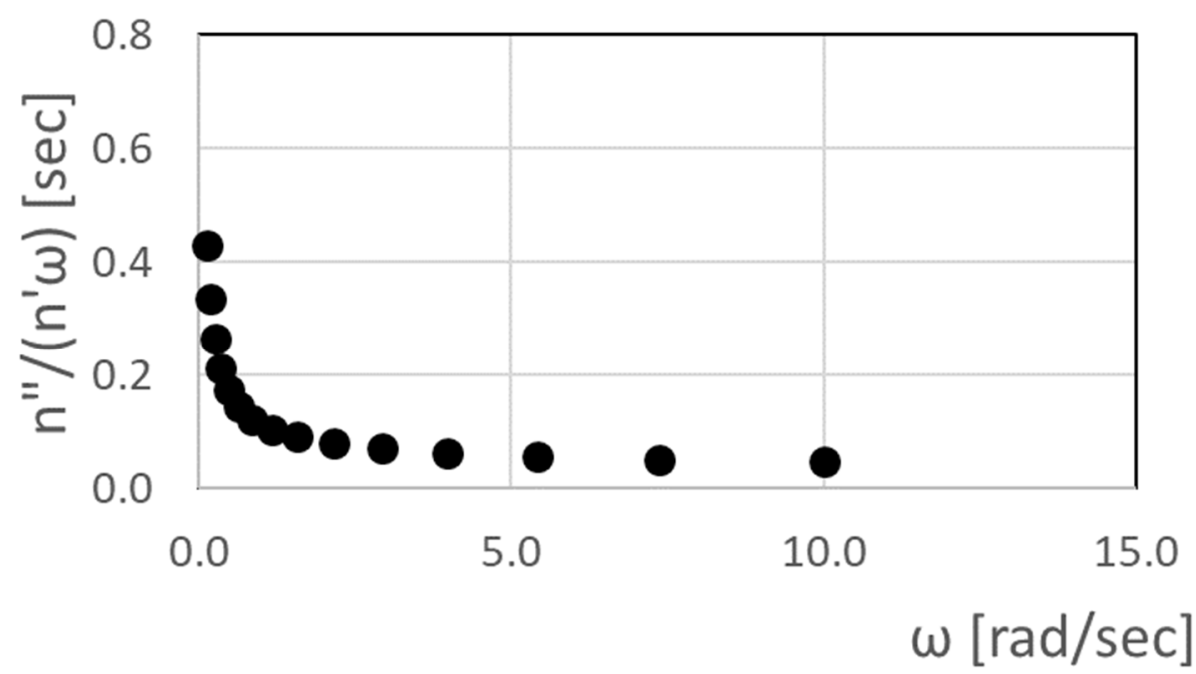

Figure 4. Complex viscosity measurement result showing dependence of $\eta^{\prime \prime} /\left(\eta^{\prime} \omega\right)$ on angular frequency from rheometer oscillatory frequency-sweep test for a solution with a polymer concentration $\rho$ of 50 ppm.

Such results indicate that polymer relaxation times from all three approaches are in approximate agreement for a polymer concentration $\rho$ of $50 \mathrm{ppm}$. 


\subsection{Prandtl Number Variation}

As polymer solutions are sufficiently dilute, the absolute viscosity $\eta$ for each concentration remains approximately constant as the shear rate varies (with the exception of shear-thinning variations near zero shear rate). The associated values of viscosity increase with polymer concentration. The resulting Prandtl numbers are determined by the equation given by

$$
\operatorname{Pr}=\frac{\eta c_{P}}{K_{f}}
$$

where $\eta$ is the absolute viscosity, $c_{P}$ is the specific heat, and $K_{f}$ is the thermal conductivity of the fluid. Figure 5 shows resulting Prandtl number values for different polymer concentrations for the initial zero shear rate condition when no disk rotation speed is employed. Within this figure, Prandtl number values are as high as 3000 , with values that generally increase with the polymer concentration. Such results illustrate effective transport and diffusion characteristics, indicating that momentum transport, quantified by the magnitude of kinematic viscosity, significantly exceeds thermal transport, quantified by the magnitude of thermal diffusivity.

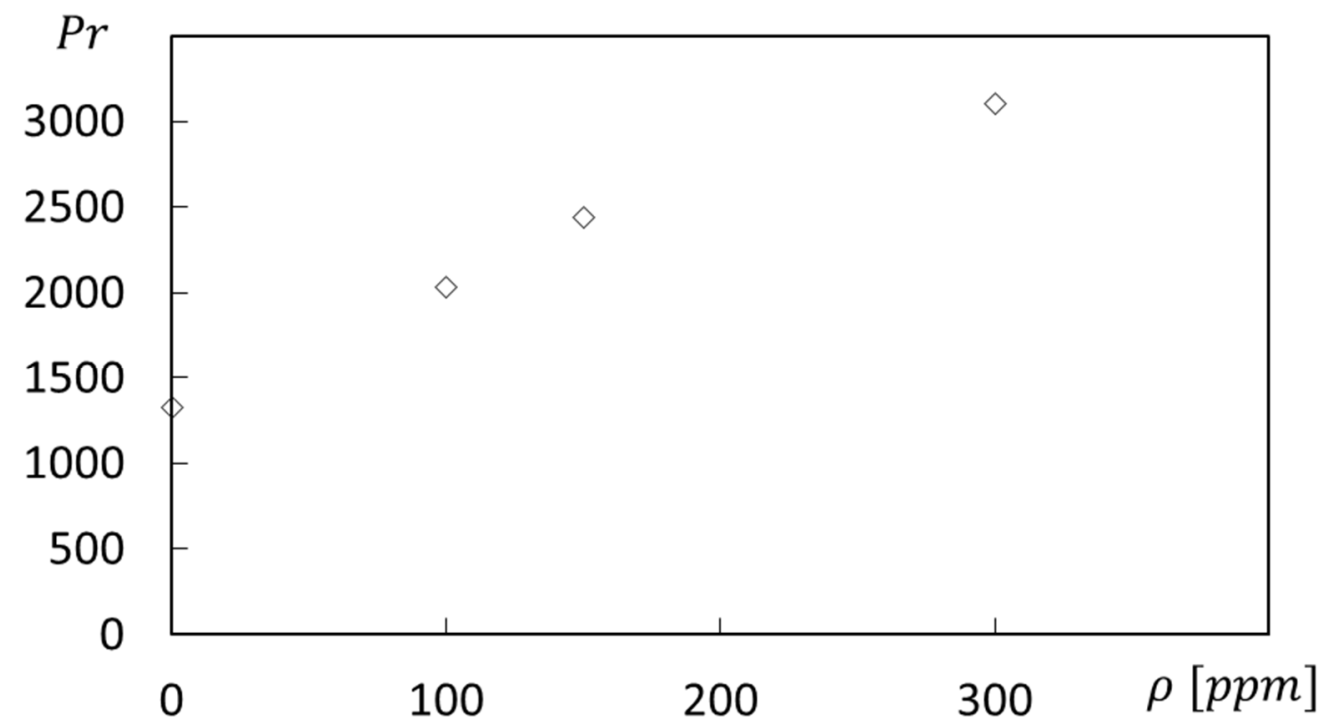

Figure 5. Prandtl number Pr variation with polymer concentration $\rho$ for zero shear rate condition $\dot{\gamma}=0$.

Another related parameter, which is discussed later in the paper, is the Schmidt number.

\subsection{Experimental Results without Advection}

Here, data are obtained with a stationary disk and no disk rotation, without the presence of flow advection. The associated data thus illustrate the influences of molecular diffusion only. Figure 6a shows data for a polymer concentration $\rho$ of $0 \mathrm{ppm}$, and Figure $6 \mathrm{~b}$ shows data for a polymer concentration $\rho$ of $150 \mathrm{ppm}$. Figure $6 \mathrm{c}$ presents scale dimensions for individual visualization images. Within the flow visualization images, the amount of radial diffusion is evidenced by the radial spreading of the dye. Note that data within these figures are provided for times as large as $90 \mathrm{~min}$. 
(a)

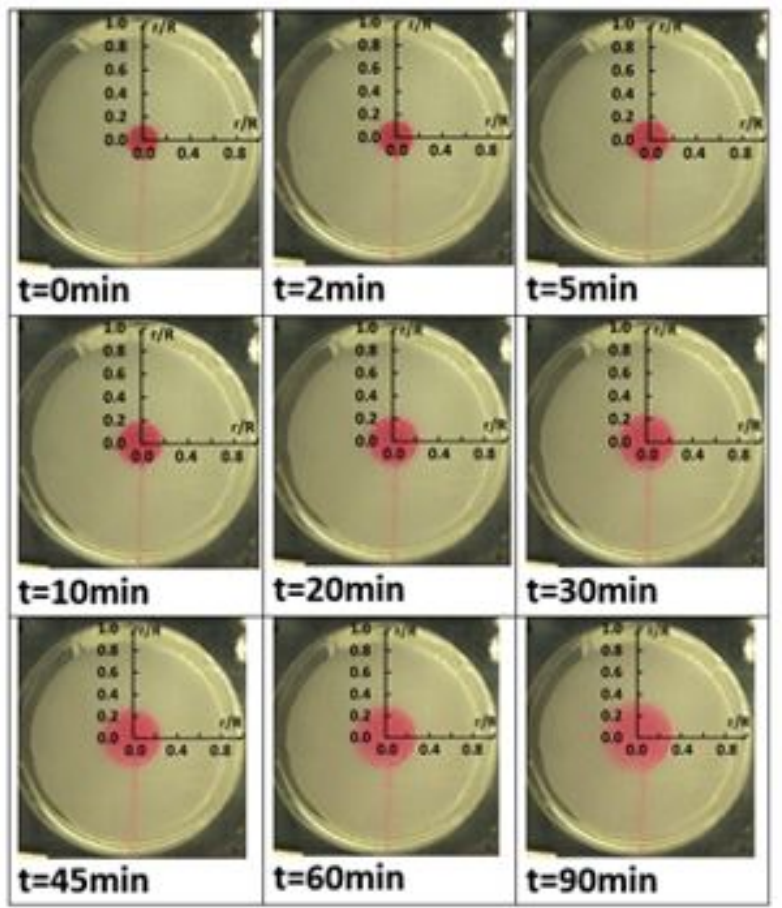

(b)

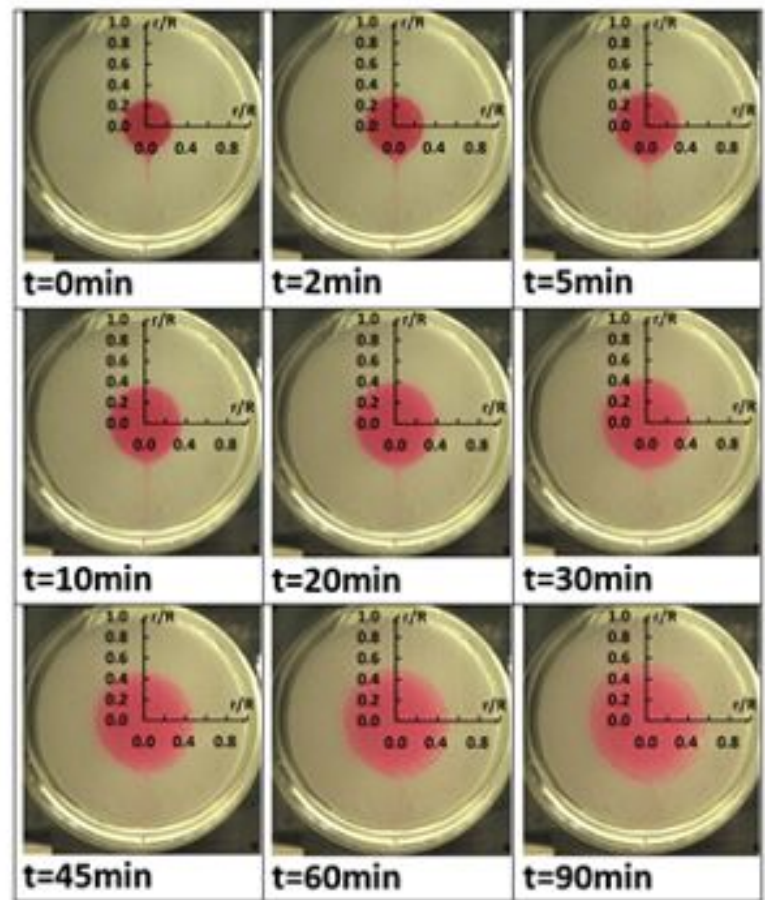

(c)

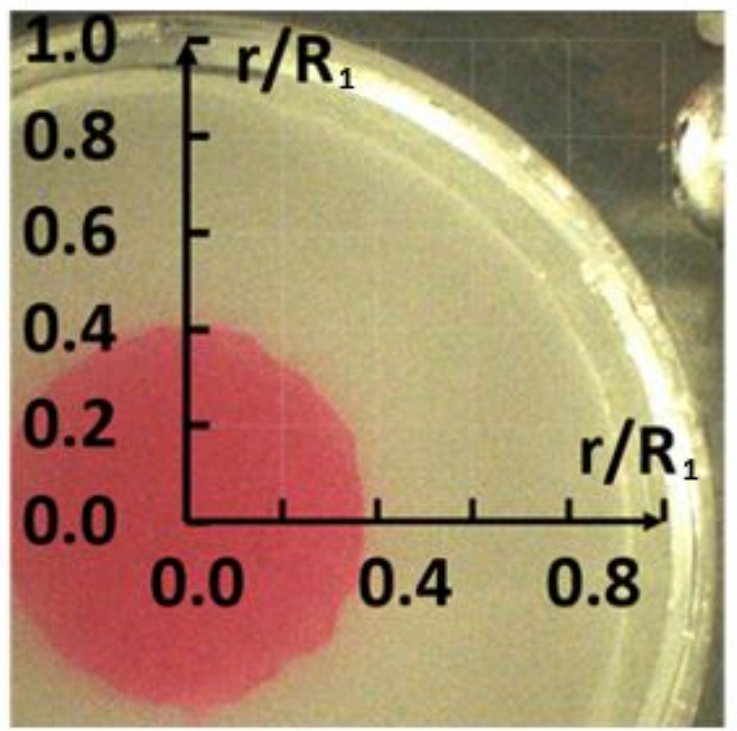

Figure 6. Time sequences of flow visualization images with no disk rotation, rotation speed $\omega$ of $0 \mathrm{rad} / \mathrm{s}$, and no circumferential advection. (a) Polymer concentration $\rho$ of 0 ppm. (b) Polymer concentration $\rho$ of $150 \mathrm{ppm}$. (c) Scale dimensions for individual visualization images.

Comparing the results in Figure $6 \mathrm{a}, \mathrm{b}$ indicates that the dye diffuses slightly more rapidly for the $150 \mathrm{ppm}$ polymer concentration, compared to the $0 \mathrm{ppm}$ sucrose solvent. Local dye concentration values are determined from local grayscale values within black and white images of the visualization data. Figure 7 shows an example of such a normalized dye concentration variation as it varies with normalized radius location, for a concentration $\rho$ of $150 \mathrm{ppm}$, a rotation speed $\omega$ of $0 \mathrm{rad} / \mathrm{s}$, and different times. Note that variations associated with the largest local gradients correspond to dye front locations within Figure $6 \mathrm{~b}$. Instantaneous dye front locations are thus identified within normalized dye concentration data at radial locations corresponding with the maximum normalized slope. Figure 8 then 
presents $\mathrm{r} / \mathrm{R}$ dye front locations as time proceeds for a polymer concentration $\rho$ of $150 \mathrm{ppm}$ and a rotation speed $\omega$ of $0 \mathrm{rad} / \mathrm{s}$. The area of the dye front, or diffusion area variation, is then determined using the equation given by

$$
A_{D}=2 \pi r H
$$

where $H$ is the fluid depth and $r$ is the value of the radial coordinate at the dye front. Figure 9 shows the resulting diffusion area $A_{D}$ variation with time for the same polymer concentration and disk rotation speed. Following recommendations on the Comsol website [12], included within this figure is a line equation, which is fit to the time-varying diffusion area experimental data.

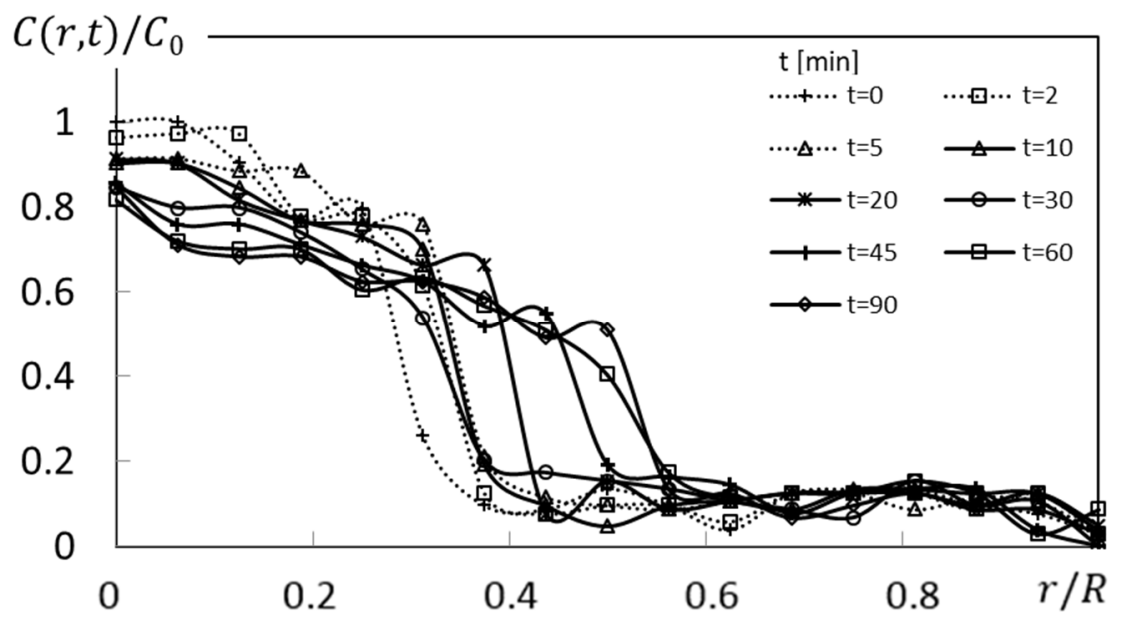

Figure 7. Normalized concentration profile variations with normalized radius location at different times $\mathrm{t}$ for polymer concentration $\rho$ of $150 \mathrm{ppm}$, rotation speed $\omega$ of $0 \mathrm{rad} / \mathrm{s}$, and no circumferential advection.

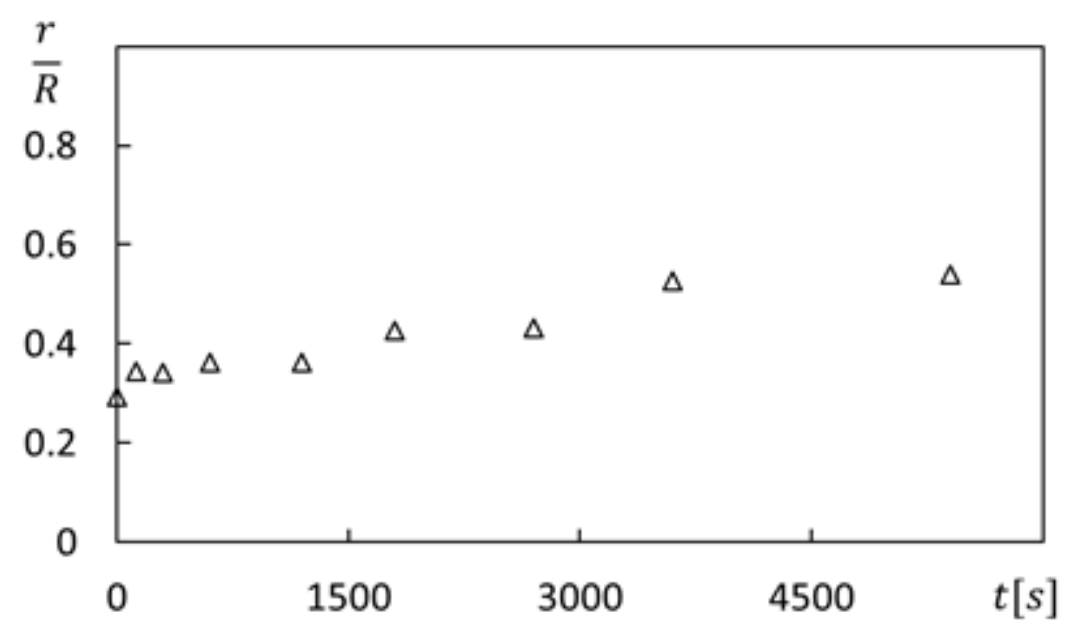

Figure 8. Dye front variation with time $t$ for polymer concentration $\rho$ of $150 \mathrm{ppm}$, rotation speed $\omega$ of $0 \mathrm{rad} / \mathrm{s}$, and no circumferential advection. 


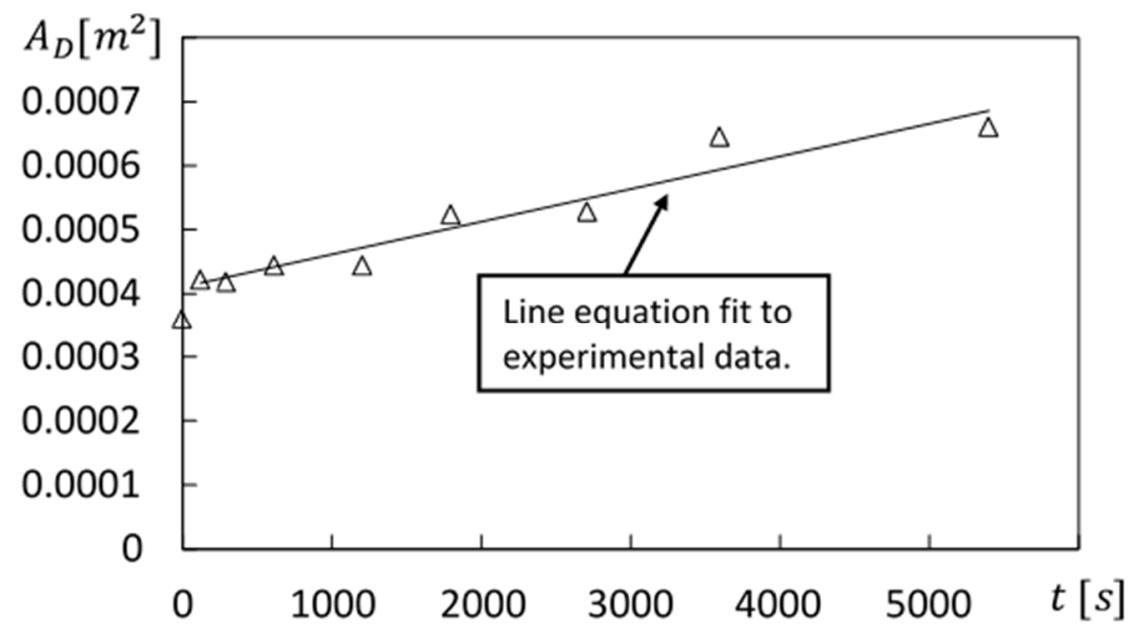

Figure 9. Diffusion area variation with time $\mathrm{t}$ for polymer concentration $\rho$ of $150 \mathrm{ppm}$, rotation speed $\omega$ of $0 \mathrm{rad} / \mathrm{s}$, and no circumferential advection.

According to Einstein [13], molecular diffusion can be considered such that "particles will pass across a unit area per unit time as the result of diffusion". The Comsol website [12] further indicates that "formally, the diffusion coefficient can be understood as parameterizing the area of a spherical surface, defined as the surface of root-mean-square displacement of material diffusing away from an infinitesimal point where a mass is initially concentrated. As the statistics of diffusion cause this area to grow linearly in time, the diffusion coefficient is a quantity described by area per time". Within the present investigation, the linear variation of diffusion area value with time is determined for different experimental conditions. The resulting molecular diffusion coefficients are then determined using the equation that is expressed using

$$
D=\alpha \frac{d A_{D}}{d t}
$$

which is referred to as the area derivative approach. Within Equation (8), the $\alpha$ factor is determined so that diffusion coefficients match values determined using other approaches.

The first of these alternative approaches is based upon solutions to Fick's second law of diffusion using a cylindrical coordinate system, as described by Lee et al. [14]. Here, diffusion coefficients are determined that provide analytically determined concentration variations with time (based upon solutions to the Fick mass diffusion equation), which match experimentally measured distributions from the present rotating Couette flow environment. This technique is referred to as the PDE approach. The second alternative approach is based upon diffusion coefficient data for fluorescein dye within a sucrose solution provided by Chenyakin et al. [15]. The third alternative approach is based upon the equation from Einstein [13], which is given by

$$
D=\frac{k T}{6 \pi R_{H D} \eta}
$$

where $D$ is the molecular diffusion coefficient and $k$ is the Boltzmann constant with a value of $1.3807 \times 10^{-23} \mathrm{~J} / \mathrm{K}$. The associated diffusing fluorescein dye results are provided for temperature $T$, absolute viscosity $\eta$, and with the effective molecule hydrodynamic radius $R_{H D}$ equal to $5.02 \times 10^{-10} \mathrm{~m} \mathrm{[15]}$.

The resulting diffusion coefficient results are given in Figure 10 for different values of polymer concentration $\rho$, as determined using each of the four methods. Note that the $\alpha$ value employed within Equation (8) to provide the area derivative approach results is $\alpha=0.0000313=3.13 \times 10^{-5}$. This value is chosen so that the area derivative results are in close agreement with values determined using the Einstein [13] and Chenyakin et al. [15] approaches. The associated diffusion coefficients are provided without any 
form of advection present, and as a result, the diffusion coefficients remain approximately constant over the range of polymer concentrations considered.

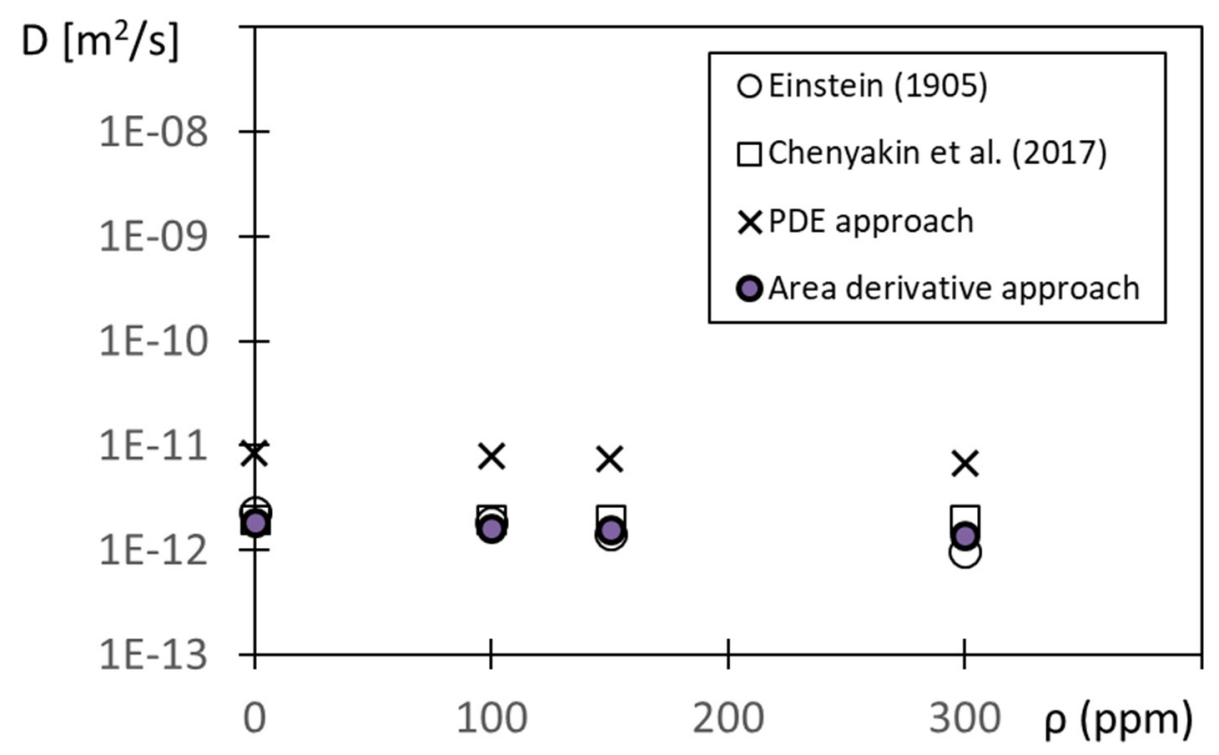

Figure 10. Diffusion coefficient variations with polymer concentration determined using different methods for rotation speed $\omega$ of $0 \mathrm{rad} / \mathrm{s}$ and no circumferential advection.

Note that the PDE approach values within Figure 10 are generally higher than the values determined using other methods. This is believed to be because the assumptions employed to obtain the associated solutions (to the Fick mass diffusion equation) are not completely consistent with the conditions associated with the present Couette flow environment.

\subsection{Experimental Results with Circumferential Advection}

Here, results are given for the testing conditions with radial diffusion and circumferential advection induced by disk rotation. Figure 11 shows time sequences of flow visualization images with a disk rotation speed $\omega$ of $1.57 \mathrm{rad} / \mathrm{s}$ with circumferential advection. Note that a rotational speed $\omega$ of $1.57 \mathrm{rad} / \mathrm{s}$ gives a shear rate $\dot{\gamma}$ of $0.721 / \mathrm{s}$ for the $R_{3}$ radial location. Figure 11a gives data for a polymer concentration $\rho$ of $0 \mathrm{ppm}$, and Figure $11 \mathrm{~b}$ gives data for a polymer concentration $\rho$ of $300 \mathrm{ppm}$. For both arrangements, fluorescein red tracer dye is used to track flow redistributions and mixing within a rotating Couette flow environment. The scale dimensions for each individual visualization image are provided in Figure 6c.

Different distributions of fluorescein red tracer dye are evident within the two instantaneous image sequences. For the $0 \mathrm{ppm}$ arrangement, dye distributions in Figure 11a continue to be concentrated near the center of the disk flow passage, even for times as large as $60 \mathrm{~s}(1 \mathrm{~min})$. Here, the dye moves radially outward only very slowly and smoothly, always with an approximately circular distribution, which evidences pure laminar flow. No variations are apparent that evidence any form of secondary flows, enhanced local mixing, or enhanced local transport. With the $300 \mathrm{ppm}$ polyacrylamide solution, dye distributions in Figure $11 \mathrm{~b}$ are then dispersed over a much larger area, relative to the center of the flow passage, with progressively increased spatial distributions, which are increasingly irregular and dispersed, as the time increases up to $60 \mathrm{~s}(1 \mathrm{~min})$. With this arrangement, the effective radial diffusion rate of the dye is notably enhanced as the flow advects through the flow passage. Such behavior evidences the presence of elastic instabilities, wherein elastic stress gradients lead to initial polymer distortion and agitation, and increased unstable polymer stretching in both the circumferential and radial directions. 
(a)

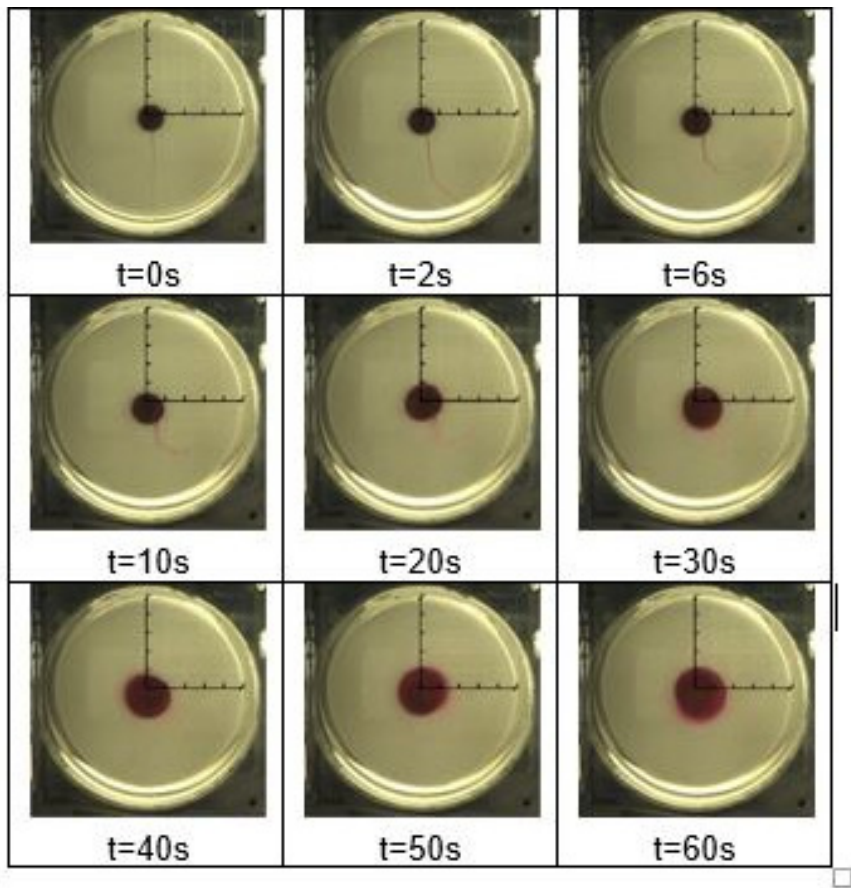

(b)

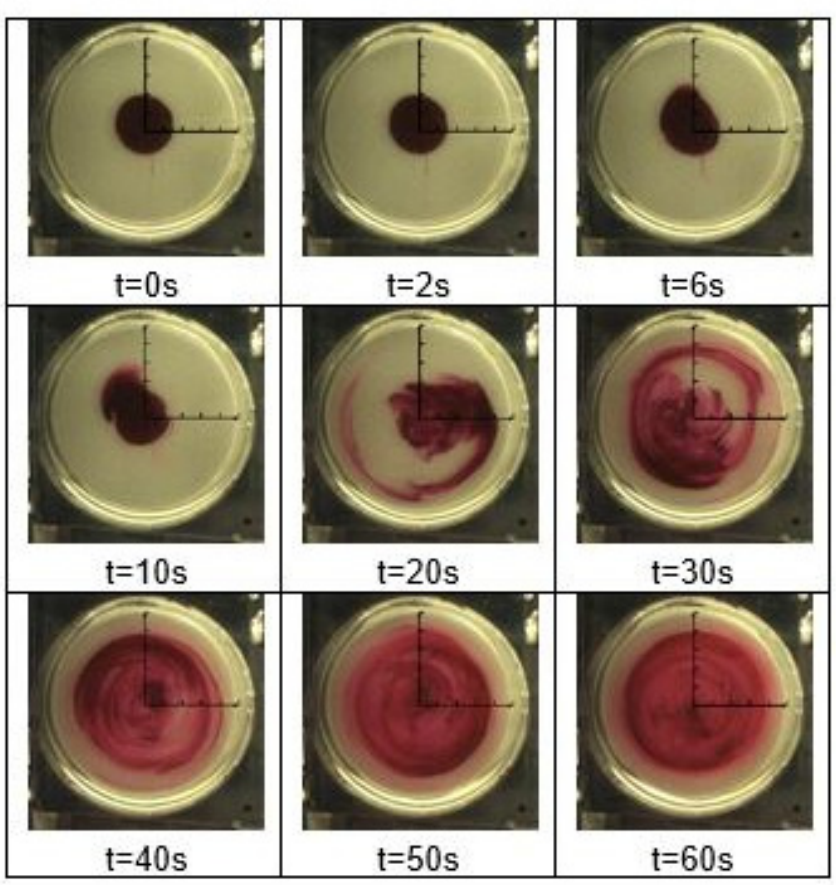

Figure 11. Time sequences of flow visualization images with disk rotation speed $\omega$ of $1.57 \mathrm{rad} / \mathrm{s}$ with circumferential advection. Scale dimensions for individual visualization images given in Figure $6 c$. (a) Polymer concentration $\rho$ of 0 ppm. (b) Polymer concentration $\rho$ of 300 ppm.

Note that effective diffusion is used as a descriptive label, to account for the presence of circumferential advection and molecular diffusion together. This point is important because polymer motions associated with elastic instabilities are not a result of true molecular diffusion or Brownian motion.

Effective diffusion coefficients $D_{\text {ef }}$ are determined from results such as those presented in Figure 11a,b, obtained with the presence of circumferential advection. The procedures, including the use of Equation (8), are the same as those employed to determine molec- 
ular diffusion coefficients using the area derivative approach. Note that the previously determined $\alpha$ value of $3.13 \times 10^{-5}$ is again utilized for this analysis.

Resulting $D_{\text {ef }}$ values are provided in Figure 12 as they vary with shear rate $\dot{\gamma}$ for different polymer concentrations $\rho$. Note that shear rate $\dot{\gamma}$ magnitudes are determined for the $\mathrm{R}_{3}$ radial location, as mentioned. Considering a shear rate $\dot{\gamma}$ of $2.871 / \mathrm{s}$, the $\mathrm{D}_{\mathrm{ef}}$ value for a polymer concentration of $300 \mathrm{ppm}$ is about 3.5 times larger than the $\mathrm{D}_{\text {ef }}$ value for a polymer concentration of $0 \mathrm{ppm}$. Such differences illustrate the enhancement due to the local development of elastic instabilities. Depending upon the specific flow conditions, $\mathrm{D}_{\mathrm{ef}}$ values with elastic instabilities present range from 30 to 700 times larger than D diffusion coefficient values associated with molecular diffusion alone.

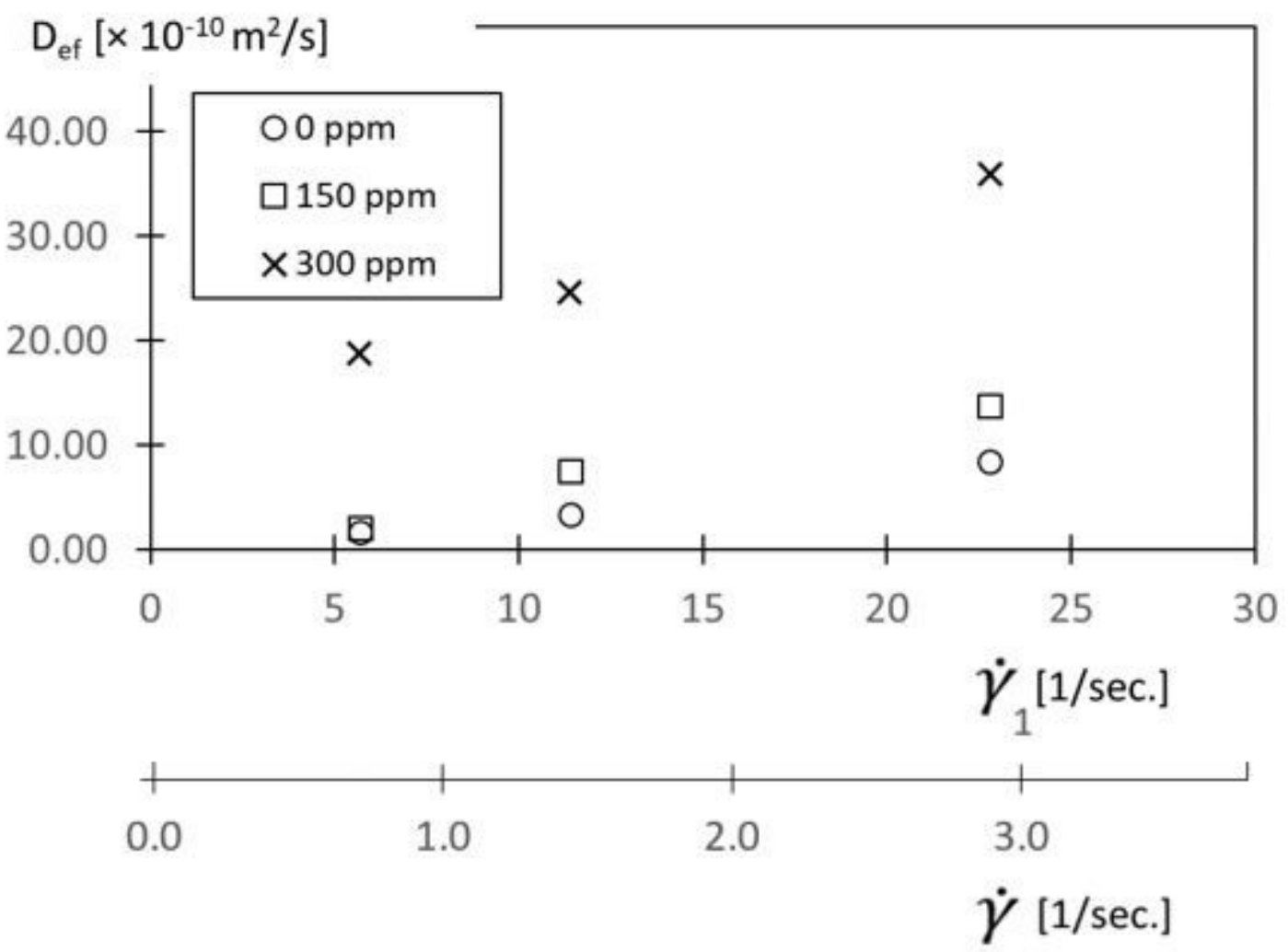

Figure 12. Effective diffusion coefficient variations with shear rate $\dot{\gamma}$ for different polymer concentrations $\rho$.

Included also within Figure 12 is a shear rate $\dot{\gamma}_{1}$ scale, which is based upon the $2 / 3 R_{1}$ radial location. Note that this scale is employed by Ligrani et al. [7] for the same flow environment and is thus provided for reference in relation to this previous investigation.

The $\dot{\gamma}$ shear rate variation, which is based upon the smallest radial location within the flow, or the $R_{3}$ radial location, is used instead within the present study because it is believed to better characterize flow conditions associated with the onset of elastic instabilities. This is because, with rotation, at any instant in time, a different shear rate is present at each radial location. As a result, the Weissenberg number value changes with radial location such that the smallest value is present at the smallest radius. Elastic instability initiation first occurs for larger shear rates at larger radii, but then propagates radially inward. The onset condition is then present at the smallest radius, which bounds the volume of fluid with developing elastic instabilities. The associated smallest radius shear rate then quantifies $\mathrm{Wi}_{\mathrm{C}}$, the critical Weissenberg number at the local onset condition.

For each shear rate, the effective diffusion coefficients in Figure 12 generally increase with polymer concentration. For each polymer concentration value, the effective diffusion coefficients generally increase with the shear rate. Such variations are due to increased advection effects, and to the associated increases in magnitudes of the local flow strain. 
As a consequence, unstable polymer stretching develops in both the circumferential and radial directions. The resulting enhanced elastic stress gradients lead to initial polymer distortion and agitation, and then to the development of elastic instabilities.

\subsection{Schmidt Number Variation}

Schmidt numbers are determined using the equation that is expressed using

$$
S c=\frac{\eta}{D_{e f} \rho_{d}}
$$

where $\eta$ is the absolute viscosity and $\rho_{d}$ is the fluid density. Figure 13 shows the Schmidt number $S c$ variation with polymer concentration $\rho$ for a shear rate of $\dot{\gamma}=2.871 / \mathrm{s}$. Note that the $D_{e f}$ values from Figure 12 are used for this determination. Within Figure 13, the $S c$ values are as high as $1.68 \times 10^{5}$, with values that generally decrease with the polymer concentration. Such results indicate that momentum transport, quantified by the magnitude of kinematic viscosity, significantly exceeds mass species transport, quantified by the magnitude of the effective diffusion coefficient. Kinematic viscosity is the absolute viscosity divided by static density.

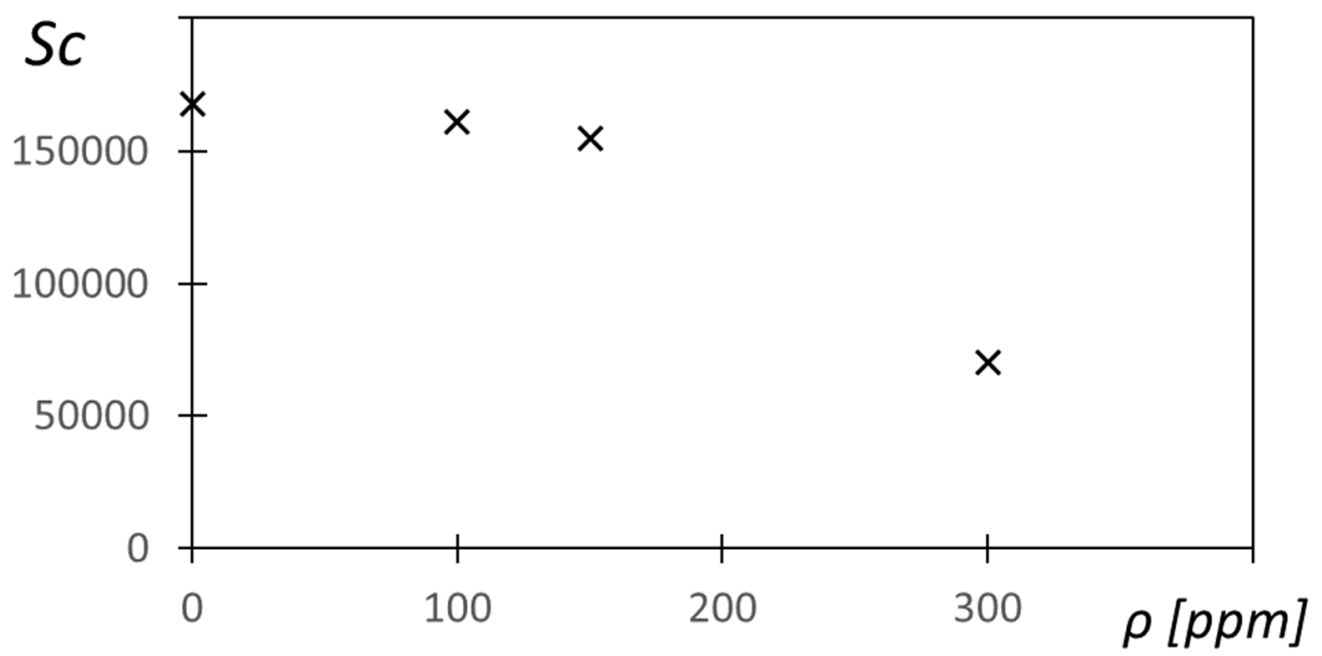

Figure 13. Schmidt number Sc variation with polymer concentration $\rho$ for a shear rate of $\dot{\gamma}=2.871 / \mathrm{s}$.

\subsection{Additional Experimental Results with Circumferential Advection}

Figure 14 shows effective diffusion coefficient variations with the Reynolds number Re for different polymer concentrations $\rho$. Considerable scatter is evident within this plot. The data point associated with the highest Reynolds number corresponds to $\rho=0$ ppm, with progressively lower Reynolds numbers as the polymer concentration $\rho$ increases. Figure 15 presents effective diffusion coefficient variations with the Weissenberg number Wi for different polymer concentrations $\rho$. Values of the elastic number associated with these data for polymer concentrations of $150 \mathrm{ppm}$ and $300 \mathrm{ppm}$ are approximately 16.5 and 28.8, respectively. Maximum values of the respective Deborah numbers for these two polymer concentrations are then approximately 11.9 and 17.6.

The values of $D_{\text {ef }}$ within Figure 15, which are associated with the onset of elastic instabilities, are in the vicinity of $10 \times 10^{-10} \mathrm{~m}^{2} / \mathrm{s}$ to $25 \times 10^{-10} \mathrm{~m}^{2} / \mathrm{s}$. This range of values is determined for $\mathrm{Wi}=\mathrm{Wi}_{\mathrm{C}}=3.8$, which is discussed in additional detail in the next section of the paper. These transition $\mathrm{D}_{\text {ef }}$ number magnitudes cover a wide range of values and are thus only in very approximate agreement with instability onset values based upon flow visualization data from Ligrani et al. [7], which are also provided for a rotating Couette flow environment. 


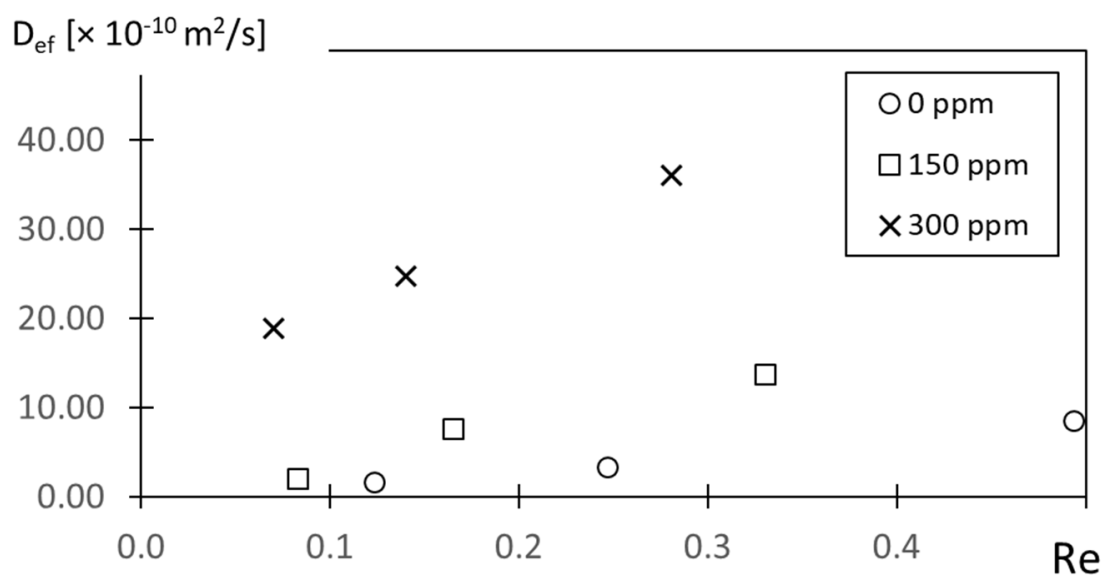

Figure 14. Effective diffusion coefficient variations with Reynolds number Re for different polymer concentrations $\rho$.

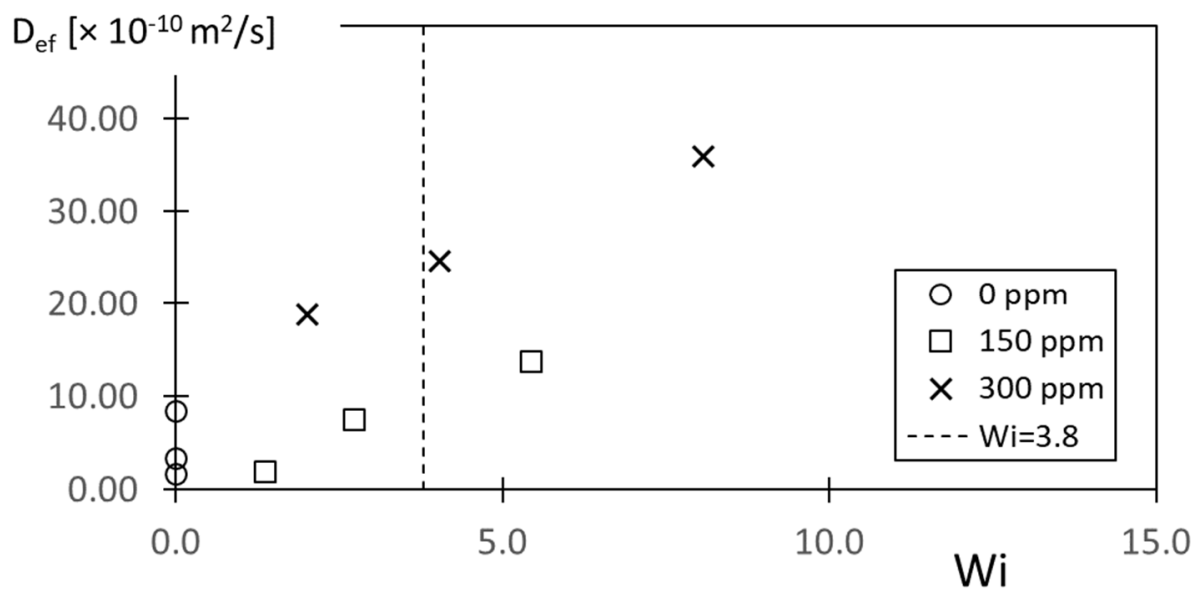

Figure 15. Effective diffusion coefficient variations with Weissenberg number Wi for different polymer concentrations $\rho$, including comparison with the Weissenberg number Wi value at the onset of elastic instability.

\subsection{Experimental Results with Circumferential Advection and the Mixing/Diffusion g Parameter}

Time-varying dye front data are also well represented using the approach of Ottino [16], as expressed using an equation given by

$$
A=A_{0}[1-\exp (-g t)]
$$

where $A=r / R_{1}, A_{0}$ is equal to $1.0, t$ is time, and $g$ is the effective mixing/diffusion rate. Larger $g$ values are thus associated with increased mass species transport, with greater local mixing for particular experimental conditions. Figure 16 shows comparisons of Equation (11) with normalized dye front variations with time $t$, provided as $r / R$ values, for different polymer concentrations. The predicted data using Equation (11) are generally in agreement with the experimental results. Parameter $g$ values are determined so that A values determined using Equation (11) for different times match the experimentally measured results. The approximate $g$ values used for this purpose are $0.0151 / \mathrm{s}$ for a polymer concentration of $0 \mathrm{ppm}, 0.041 / \mathrm{s}$ for a polymer concentration of $80 \mathrm{ppm}, 0.061 / \mathrm{s}$ for a polymer concentration of $100 \mathrm{ppm}, 0.141 / \mathrm{s}$ for a polymer concentration of $150 \mathrm{ppm}$, and $0.151 / \mathrm{s}$ for a polymer concentration of $300 \mathrm{ppm}$. Here, magnitudes of $A=r / R$ generally increase with polymer concentration for each time, which means that fluorescein dye distributions are more spread out, to cover larger volumes, due to locally increased effective diffusion. 


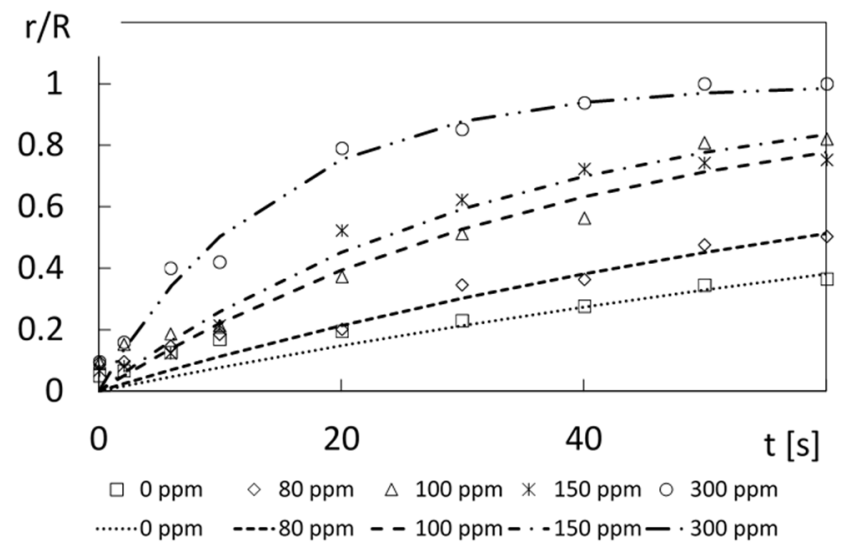

Figure 16. Dye front variation with time $t$ for different polymer concentrations $\rho$ and rotation speeds $\omega$ of $6.29 \mathrm{rad} / \mathrm{s}$ with circumferential advection, including comparisons with mixing/diffusion rate $g$ parameter Equation (11) from Ottino [16].

Figure 17 then shows mixing/diffusion rate g parameter variations with rotation speed for different polymer concentrations. Magnitudes of $g$ effective diffusion rate generally increase with disk rotation speed. Mixing/diffusion rate g value increases are also associated with larger polymer concentrations for each rotational speed considered. Within this figure, the $g$ value associated with the onset of elastic instabilities is indicated as $\mathrm{g}=0.04 \mathrm{1} / \mathrm{s}$. This value is in agreement with onset conditions for flow visualization data from Ligrani et al. [7], especially for larger shear rate magnitudes.

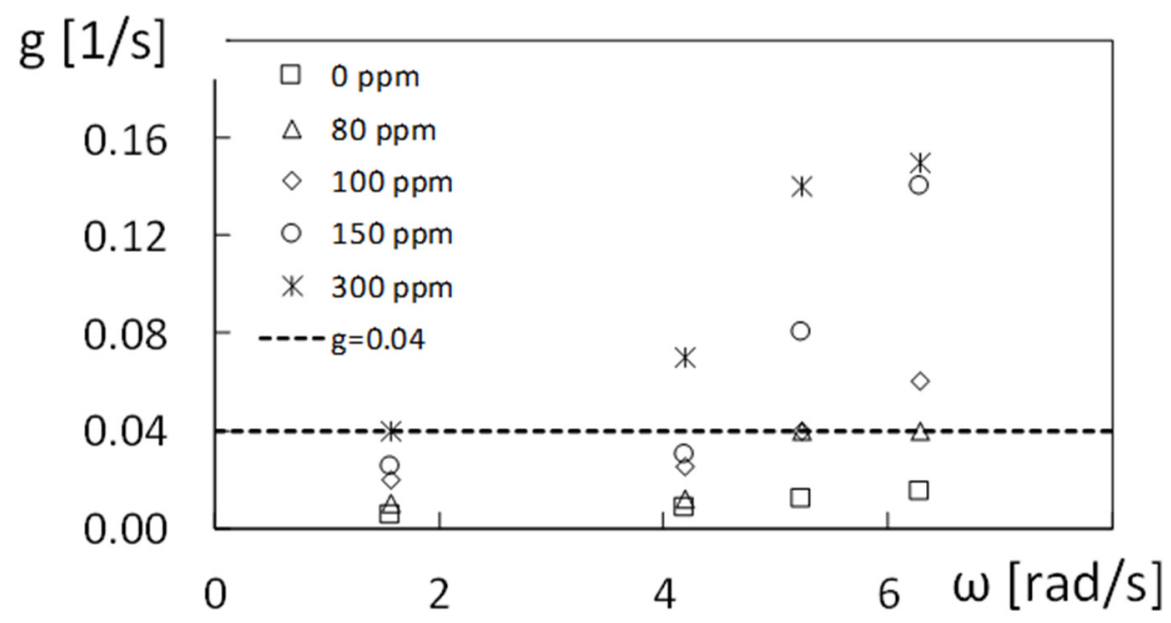

Figure 17. Mixing/diffusion rate g parameter variations with rotation speed $\omega$ for different polymer concentrations $\rho$, including comparison with the mixing/diffusion rate $g$ parameter value at the onset of elastic instability.

Figure 18 shows mixing/diffusion rate g parameter variations with Reynolds number Re for different polymer concentrations $\rho$. Included is the value of the mixing/diffusion rate g parameter of $0.041 / \mathrm{s}$ for the onset of elastic instability from Ligrani et al. [7]. Within this figure, much data scatter is present, where the data point associated with the highest Reynolds number is obtained with a polymer concentration $\rho=0 \mathrm{ppm}$. 


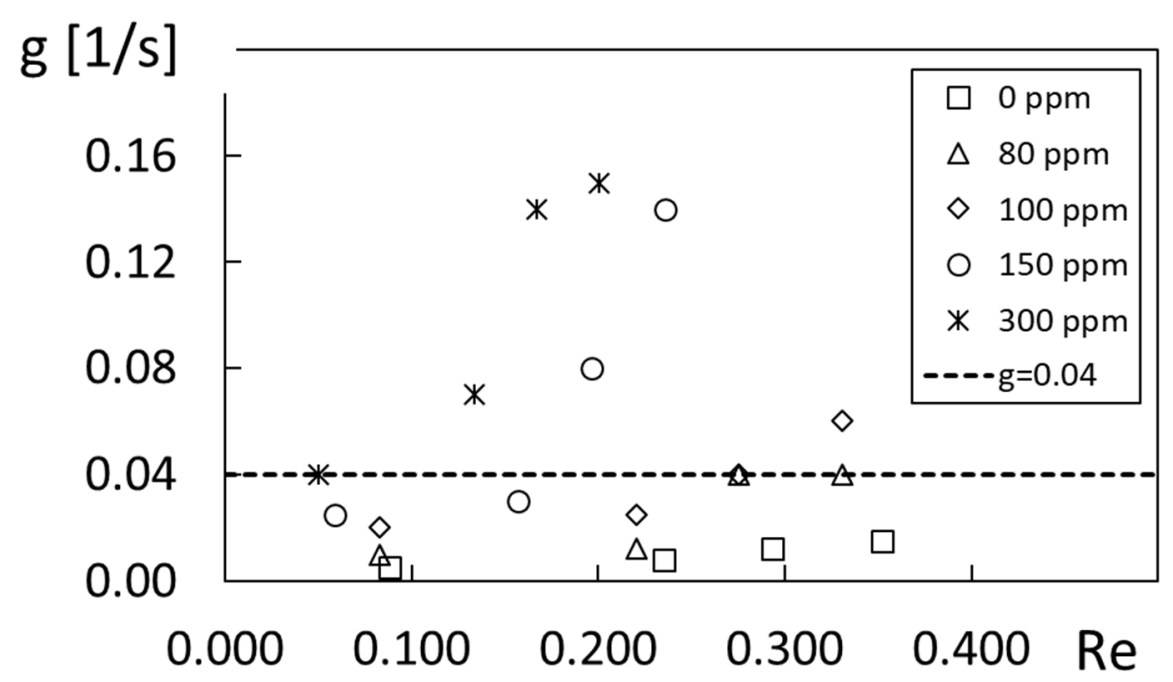

Figure 18. Mixing/diffusion rate g parameter variations with Reynolds number Re for different polymer concentrations $\rho$, including comparison with the mixing/diffusion rate $g$ parameter value at the onset of elastic instability.

Mixing/diffusion rate $\mathrm{g}$ parameter variations are given in Figure 19 for different polymer concentrations $\rho$ as they vary with the Weissenberg number Wi. Here, data for different $\rho$ values collect in an approximate manner into a single distribution. Note that Figure 19 also identifies the g parameter value of 0.041 /s at the onset of elastic instability [7], and $\mathrm{Wi}_{\mathrm{c}}$, the critical Weissenberg number value, of 3.8 also at the onset of elastic instability. As these two parameter specifications are consistent with each other and with the flow visualization data of Ligrani et al. [7], appropriate characterization is provided of the conditions associated with the onset of elastic instabilities. As such, these approaches provide more physically appropriate representations, compared to characterizations that are based upon variations in $\mathrm{D}_{\text {ef }}$, the effective diffusion coefficient. Note that the shear rate, Weissenberg number, and Reynolds number associated with the data in Figures 18 and 19 are all determined for the $\mathrm{R}_{3}$ radial location. Note also that the $\mathrm{Wi}_{\mathrm{C}}$ value of 3.8 is consistent with elastic instability onset characterizations from other sources, which are generally associated with Wi values from 2.0 to 5.0 [6,11].

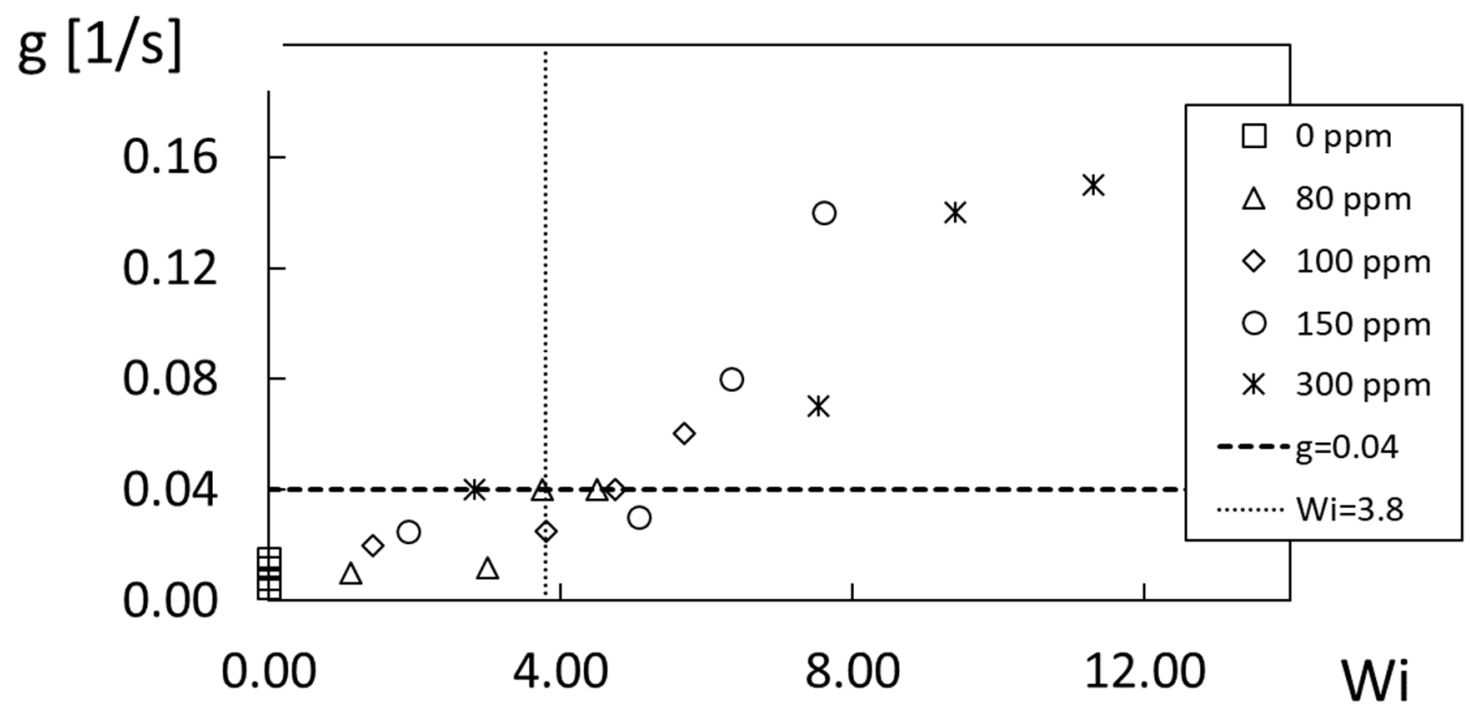

Figure 19. Mixing/diffusion rate g parameter variations with Weissenberg number Wi for different polymer concentrations $\rho$, including comparison with the mixing/diffusion rate $g$ parameter value at the onset of elastic instability, and comparison with the Weissenberg number Wi value at the onset of elastic instability. 


\section{Summary and Conclusions}

We considered effective diffusion, characterized by magnitudes of effective diffusion coefficients, in order to quantify mass transport due to the onset and development of elastic instabilities. Effective diffusion coefficient magnitudes were determined using different analytic approaches, as they were applied to tracked visualizations of fluorescein dye front variations, as circumferential advection was imposed upon a flow environment produced using a rotating Couette flow arrangement. Effective diffusion coefficient results were provided for a range of flow shear rates, which were produced using different Couette flow rotation speeds and two different flow environment fluid depths. To visualize the flow behavior within the rotating Couette flow environment, minute amounts of fluorescein dye were injected into the center of the flow container using a syringe pump. This dye was then redistributed within the flow by radial diffusion only when no disk rotation was used, and by radial diffusion and by circumferential advection when disk rotation was present. Associated effective diffusion coefficient values, for the latter arrangement, were compared to coefficients values with no disk rotation, which were compared to molecular diffusion alone, in order to quantify enhancements due to elastic instabilities. Experiments were conducted using viscoelastic fluids, which were based on a $65 \%$ sucrose solution, with different polymer concentrations ranging from $0 \mathrm{ppm}$ to $300 \mathrm{ppm}$. Associated Reynolds numbers based on fluid depth and radial flow velocity ranged from 0.00 to 0.50 .

Significant changes to flow characteristics were present due to the onset and development of elastic instabilities. The resulting effective diffusion coefficients $\mathrm{D}_{\text {ef }}$ for a polymer concentration of $300 \mathrm{ppm}$ were as much as 3.5 times larger than the effective diffusion coefficients $D_{\text {ef }}$ associated with a polymer concentration of $0 \mathrm{ppm}$. Note that this comparison was made for the same local shear rate and disk rotation speed. Such differences illustrate effective diffusion transport enhancements due to the local development of elastic instabilities. Depending upon the specific flow conditions, $D_{\text {ef }}$ values, with elastic instabilities present, ranged from 30 to 700 times larger than D diffusion coefficient values associated with molecular diffusion alone. As such, variations in the effective diffusion coefficients illustrated changes to local mass transport magnitudes, which were associated with the onset and development of elastic instabilities. For example, for each rotation speed and shear rate, the effective diffusion coefficients generally increased with polymer concentration. For each polymer concentration value, the effective diffusion coefficients generally increased with rotation speed and shear rate.

Mixing/diffusion rate g parameter variations for different polymer concentrations $\rho$ and for different Weissenberg numbers Wi evidenced the conditions associated with the onset of elastic instabilities. This onset condition was characterized by a $g$ parameter value of $0.041 / \mathrm{s}$ and by a critical Weissenberg number $\mathrm{Wi}_{\mathrm{c}}$ value of 3.8. As these two parameter specifications were consistent with each other and with the flow visualization data of Ligrani et al. [7], appropriate characterization was provided of the conditions associated with the onset elastic instabilities. As such, these representations are more physically appropriate, compared to characterizations that are based upon variations in $\mathrm{D}_{\text {ef }}$, the effective diffusion coefficient.

Author Contributions: Conceptualization, P.L.; methodology, P.L.; software, M.S.; validation, M.S. and V.H.; formal analysis, M.S. and V.H.; investigation, P.L., M.S. and V.H.; resources, P.L.; data curation, P.L.; writing-original draft preparation, P.L., M.S. and V.H.; writing—review and editing, P.L., M.S. and V.H.; visualization, M.S. and V.H.; supervision, P.L.; project administration, P.L.; funding acquisition, P.L. All authors have read and agreed to the published version of the manuscript.

Funding: The research described in this paper is supported by the U.S. National Science Foundation, Grant No. CBET-1501587.

Data Availability Statement: Data from the present research effort are available upon request.

Acknowledgments: M. Snell, G. Liverett, D. Copeland, C. Ren, J. Terrell, and S. Naderian are acknowledged for their excellent laboratory activities in acquiring and analyzing a portion of the 
experimental results that are presented within the present paper. The Anton Paar Corporation is acknowledged for their guidance and support in regard to our use of a commercial Anton Paar Rheometer MCR 302 during the present investigation.

Conflicts of Interest: The authors declare no conflict of interest.

\section{Nomenclature}

$\begin{array}{ll}\mathrm{A}_{\mathrm{D}} & \text { Dye front area } \\ \mathrm{C}_{\mathrm{o}} & \text { Initial local dye concentration at } \mathrm{t}=0 \text { and } \mathrm{r}=0 \\ \mathrm{C}(\mathrm{r}, \mathrm{t}) & \text { Local dye concentration } \\ \mathrm{C}_{\mathrm{p}} & \text { Specific heat } \\ \mathrm{D} & \text { Molecular diffusion coefficient } \\ \mathrm{D}_{\mathrm{ef}} & \text { Effective diffusion coefficient } \\ \mathrm{Deb} & \text { Deborah number } \\ \mathrm{El} & \text { Elastic number } \\ \mathrm{g} & \text { Effective mixing/diffusion rate } \\ \mathrm{G}^{\prime} & \text { Elastic modulus } \\ \mathrm{G}^{\prime \prime} & \text { Viscous modulus } \\ \mathrm{H} & \text { Fluid depth } \\ \mathrm{K}_{\mathrm{f}} & \text { Thermal conductivity of the fluid } \\ \mathrm{Pr} & \text { Prandtl number } \\ \mathrm{r} & \text { Radial coordinate } \\ \mathrm{R}_{1} & \text { Outer radius of disk } \\ \mathrm{R}_{2} & \text { Inner radius of cup flow container } \\ \mathrm{R}_{3} & \text { Outer radius of rotating shaft } \\ \mathrm{R}_{\mathrm{HD}} & \text { Effective molecule hydrodynamic radius } \\ \mathrm{Re} & \text { Reynolds number } \\ \mathrm{Sc} & \text { Schmidt number } \\ \mathrm{t} & \text { Time } \\ \mathrm{T} & \text { Absolute temperature } \\ \mathrm{u} & \text { Local circumferential fluid velocity } \\ \mathrm{Wi} & \text { Weissenberg number } \\ \mathrm{Wi} \mathrm{i}_{\mathrm{c}} & \text { Critical Weissenberg number } \\ \mathrm{x} & \text { First lateral coordinate } \\ \mathrm{y} & \text { Second lateral coordinate } \\ \mathrm{z} & \text { Normal coordinate } \\ \theta & \text { Circumferential coordinate } \\ \eta & \text { Absolute viscosity } \\ \eta^{\prime} & \text { In-phase viscosity } \\ \eta^{\prime \prime} & \text { Out-of-phase viscosity } \\ \eta_{0} & \text { Absolute viscosity at zero shear rate } \\ \eta_{\infty} & \text { Absolute viscosity at infinite shear rate } \\ \rho & \text { Polymer concentration } \\ \rho_{\mathrm{d}} & \text { Fluid density } \\ \dot{\gamma}_{1} & \text { Local fluid shear rate based upon } \mathrm{R}_{3} \\ \lambda & \text { Pocal fluid shear rate based upon } 2 / 3 \mathrm{R}_{1} \\ \mathrm{w} & \text { Angular rotation speed } \\ & \end{array}$

\section{References}

1. James, D.F. Boger fluids. Annu. Rev. Fluid Mech. 2009, 41, 129-142. [CrossRef]

2. Groisman, A.; Steinberg, V. Elastic vs. inertial instability in a polymer solution flow. Europhys. Lett. 1998, 43, 165-170. [CrossRef]

3. Groisman, A.; Steinberg, V. Elastic turbulence in curvilinear flows of polymer solutions. New J. Phys. 2004, 6, 312-319. [CrossRef]

4. Burghelea, T.; Segre, E.; Bar-Joseph, I.; Groisman, A.; Steinberg, V. Chaotic flow and efficient mixing in a microchannel with a polymer solution. Phys. Rev. E 2004, 69, 066305. [CrossRef] [PubMed]

5. Burghelea, T.; Segre, E.; Steinberg, V. Elastic turbulence in von Karman swirling flow between two disks. Phys. Fluids 2007, 19, 053104. [CrossRef] 
6. Whalley, R.D.; Abed, W.M.; Dennis, D.J.C.; Poole, R.J. Enhancing heat transfer at the micro-scale using elastic turbulence. Theor. Appl. Mech. Lett. 2015, 5, 103-106. [CrossRef]

7. Ligrani, P.M.; Su, M.; Pippert, A.; Handler, R.A. Thermal transport of viscoelastic fluids within rotating Couette flows. AIAA J Thermophys. Heat Transf. 2020, 34, 121-133. [CrossRef]

8. Zimm, B.H. Dynamics of polymer molecules in dilute solution: Viscoelasticity, flow birefringence and dielectric loss. J. Chem. Phys. 1956, 24, 269-278. [CrossRef]

9. Bohme, H. Non-Newtonian Fluid Mechanics. In North-Holland Series in Applied Mathematics and Mechanics; North-Holland Publishing Company: Amsterdam, The Netherlands, 1987.

10. Sunthar, P. Polymer rheology. In Rheology of Complex Fluids; Springer: New York, NY, USA, 2010; pp. 171-191.

11. Yao, G.; Yang, H.; Zhao, J.; Wen, D. Experimental study on flow and heat transfer enhancement by elastic instability in swirling flow. Int. J. Therm. Sci. 2020, 157, 106504. [CrossRef]

12. Comsol Website. 2018. Available online: https://www.comsol.com/multiphysics/diffusion-coefficient (accessed on 15 September 2021).

13. Einstein, A. Über die von der molekularkinetischen Theorie von der Wärme geförderten Bewegung von in ruhenden Flüssigkeiten suspendierten Teilchen/On the motion of small particles suspended in liquids at rest requires by the molecular-kinetic theory of heat. Ann. Der Phys. 1905, 17, 549-560. [CrossRef]

14. Lee, S.; Lee, H.-Y.; Lee, I.-F.; Tseng, C.-Y. Ink diffusion in water. Eur. J. Phys. 2004, 25, 331-336. [CrossRef]

15. Chenyakin, Y.; Ullmann, D.A.; Evoy, E.; Renbaum-Wolff, L.; Kamal, S.; Bertram, A.K. Diffusion coefficients of organic molecules in sucrose-water solutions and comparison with Stokes-Einstein equation. Atmos. Chem. Phys. 2017, 17, 2423-2435. [CrossRef]

16. Ottino, J.M. The Kinematics of Mixing: Stretching, Chaos and Transport; Cambridge University Press: Cambridge, UK, 1989. 